\title{
EFFECTS OF HIGH FREQUENCY VIBRATION ON CRITICAL MARANGONI NUMBER
}

\author{
H. Tang and W. R. Hu \\ Institute of Mechanics, Chinese Academy of Sciences, Beijing I00080, China
}

\begin{abstract}
The influence of vibration on thermocapillary convection and critical Marangoni number in liquid bridge of half floating zone was discussed for the low frequency range 0.4-1.5 $\mathrm{Hz}$ and the intermediate frequency range $2.5-15 \mathrm{~Hz}$ in our previous papers. This paper extends the study to high frequency range $15-100 \mathrm{~Hz}$. This ground based experiment was completed on the deck of an electromagnetic vibration machine. The results of our experiment shows when the frequency of the applied acceleration is high enongh, the amplitude of the time varying part of the temperature response is disappear and the shape of the free surface of the liquid bridge exhibits no flactuations due to inertia. The critical Marangoni number which is defined to describe the transitions from a peroidical convection in response to vibration to an oscillatory convection due to internal instability is nearly the same as the critical Marangoni number for oscillatory flow in the absence of vibration.
\end{abstract}

\section{INTRODUCTION}

In low gravity emvironment, surface tension forces will play a dominant role in driving fluid flow. The convection driven by surface tension may become oscillatory if the applied temperature difference between the maximum at middle and melt temperature at both rods in the liquid bridge exceeds a critical value/1,2/. A lot of experiments and many numerical simnlations have been conducted $/ 3 /$. There have several suggestions to explain the occurrence of such oscillatory convection $/ 4,5 /$, but the problem is still open.

In a spacecraft, there are residual acceleration with variable magnitnde and oritentation. In recent years, there has been a growing interest in identifying the spacecraft vibration environment and the effects of residual acceleration on the results of space experiments. For example, Charles R. Bangher concluded low frequency vibration environment for five shattle Missions/6/, J.I.D. Alexander analysed the sensitivity of a liquid bridge/7/. In onr previous paper $/ 8,9 /$, the effects of vibration on critical Marangoni number were analyzed in the low frequency range 0.4-1.5 Hz and intermediate frequency range 3-15 $\mathrm{Hz}$. It is common knowledge that depending on the frequency, vibration will have different effects on fluid flow and its instability. In the present paper, the infuence of vibration with higher frequency range, i.e $15-100 \mathrm{~Hz}$, on critical Marangoni number is studied.

\section{EXPERIMENTAL}

The vibration was achieved by high frequency electromagnetic vibration machine. The orientation of the vibration is parallel to the acceleration of steady earth gravity, The strength $\Delta g$ and the frequency $f$ of this machine can be adjusted from $0.1 \mathrm{~g}$ to $2 \mathrm{~g}, 10$ to $1000 \mathrm{~Hz}$ respectively. A half floating zone was fixed on the deck of vibration machine. 
The scheme is shown in Fig. 1. Experiment medium of 10 cst silicon oil was filled in the gap between the upper and lower coaxial rods. The upper rod is heated by the electric resistance wires to establish the temperature difference across liquid. The temperatures of upper and lower rods were measured by thermal conples. Another thermal conple was inserted in the liquid bridge through lower rod to measure the temperature variation at a fixed point(see fig. 1) in the liquid. A He-Ne laser was used to obtain a light sheet of $0.3 \mathrm{~mm}$ in thickness throngh a vertical cross section of the liquid bridge. The trajectories of tracing particles were recorded continuonsly by a COD camera.

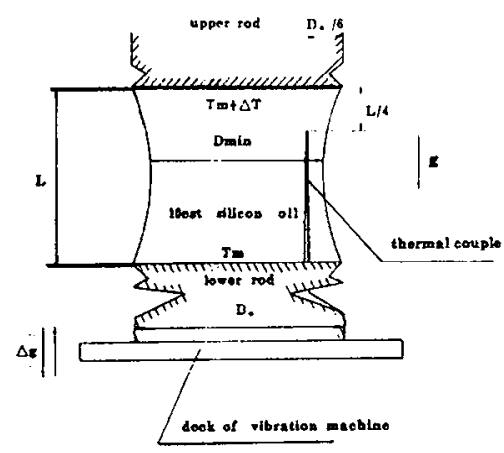

Fig. 1 Scheme of liquid bridge

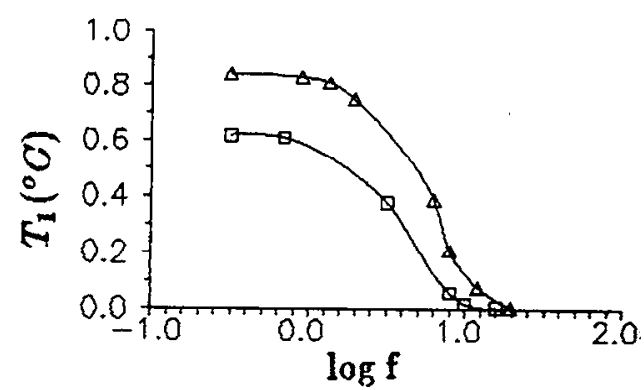

Fig. 2 Relation betwwen the ampiltude of the time varying temperature and vibration at a fixed temperature difference $\Delta T=40^{\circ} \mathrm{C}$. Here $\square: g^{*}=0.16, \Delta: g^{*}=0.25$

The diameter $D_{0}$ and height $l$ of liqnid bridge are $3.0 \mathrm{~mm}$ and $2.4 \mathrm{~mm}$ respectively, and the Bond number is defined as

$$
B_{0}=\rho g \beta l^{2} /|d \sigma / d \tau|
$$

where $\rho, \tau, \sigma$ are, respectively, the density, temperature and surface tenwsion. $\beta$ is the thermal expansion. In this case, $B_{0}=0.58$ and the effects of surface tension dominate in comparsion to the buoyancy. Many relative works have indicated that the critical parameters include the volume of liquid bridge measured by the ratio of minmumn diameter $D$ min to the rod diameter $D_{0}$ as well as Marangoni number $/ 10 \%$. In our experiment we adopt the ratio $D \mathrm{~min} / D_{0}=0.72$ and introduced a dimensionaless amplitude of the applied acceleration $g^{*}=|\Delta g| / g_{0}$. For convenience, we denote the periodical convection generally in response to applied acceleration as $\mathrm{PC}$ and the oscillatory convection induced by internal instibility as $\mathrm{OC}$.

\section{EPFECTS OP VIBRATION ON OSCILLATORY CONVECTION}

Considering the joint action of the applied temperature difference $\Delta T$ and the applied acceleration. The temperature at the fixed point in liquid bridge measured by thermal couple may be expressed as

$$
T=T_{0}+T_{1} \sin \left(\omega_{1} t+\Psi_{1}\right)
$$

where $T_{0}$ is the mean part of temperature, $T_{1}$ and $\omega_{1}$ are, respectively, the amplitude and frequency of the time varying part of the temperature response induced by an applied acceleration.

Fig. 2 shows the relation between the amplitude of the time varying part of the temperature response and the ferquency of the applied acceleration at a fixed temperature $\Delta T=40^{\circ} \mathrm{C}$. It shows that the amplitude of the time varying part of the temperature response almost vanish when the ferquency of the applied accceleration is higher than 
$15 \mathrm{~Hz}$. In the high frequency range, the orientation of the applied acceleration varies too quirkly and the inertia of the liquid makes the time varying part of the temperature response vanish.

If the Marangoni number in the liquid bridge is larger than a critical vaule, a transition from steady flow to an oscillatory one occurs. There's also a transition from PC to OC when the system is subject to vibration. Our previous paper indicated that for both low and intermediate frequency, vibration can influence the critical Marangoni number, The internal instibility makes the flow field asymmetric, The distorted temperature field results from a superposition of the time varying temperature response to applied acceleration and internal temperature oscillations caused by thermocapillary instability. Because in the HF range, the time varying part of the temperature response almost vanishes, the appearance of low frequency temperature oscillations (see Fig. 3) and an asymmetric flow field(see Fig. 4) when subject to high frquency vibration were used as the criterion for determining whether there had thermocapillary oscillatory convection in liquid bridge. We could deduce the effects of vibration on critical Marangoni number by changing the strength $g^{*}$ and frequency $f$ of applied acceleration.

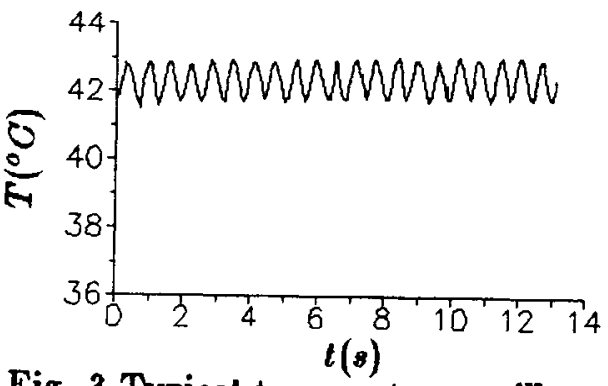

Fig. 3 Typical temperature oscillatory curve in high frequency vibration environment for $\mathrm{g}^{*}=0.5, \mathrm{f}=40 \mathrm{~Hz}$ $\Delta T=65^{\circ} \mathrm{C}$

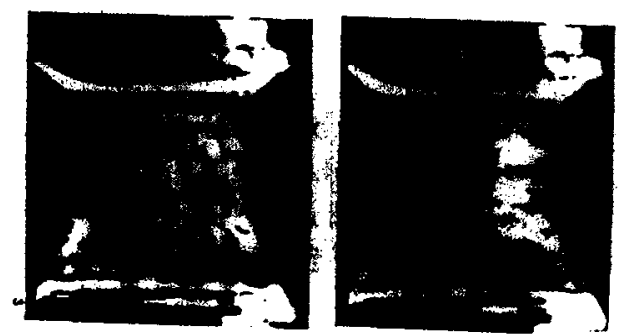

Fig. 4 Typical symmetric $\left(\Delta T=40^{\circ} C\right.$, left $)$ and asymmetric $\left(\Delta T=68^{\circ} \mathrm{C}\right.$, right) flow pattern for high frequency vibration $\left(f=50 \mathrm{~Hz}, \mathrm{~g}^{*}=0.5\right)$

Fig. 5 shows the relation between the critical Marangoni number and the frequency of the applied vibration at fixed amplitude. We can find that, at high frequency, the critical Marangoni number will decrease relative to that for intermediate frequency when the vibration amplitude is fixed. The frequency at which the critical Marangoni number is a maximum depends on the vibration amplitade. The larger the vibration amplitude is, the higher this frequency will be. When the frequency is high enough, the critic al Marangoni number will approach that for the case of zero applied vibration. Fig. 6 shows the relation between critical Marangoni number and vibration amplitude. For high frequency, we

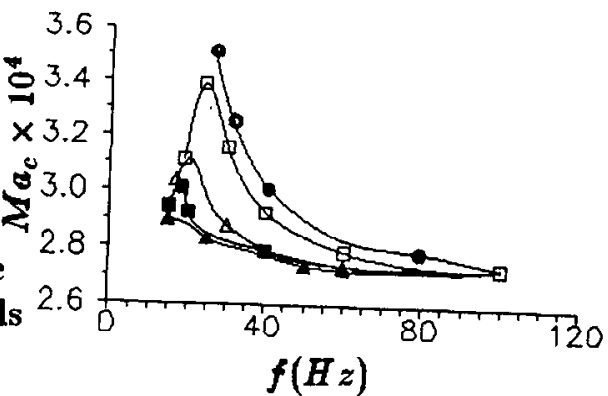

Fig. 5 Relation between the critical Marangoni number and the vibration frequency at fixed amplitude. Here $\Delta: g^{*}=0.15, \mathrm{a}: \mathrm{g}^{*}=0.2, \Delta: \mathrm{g}^{*}=0.4$, $\square: g^{*}=0.5, O: g^{*}=0.6$. find that the critical Marangoni number will slightly increase with increasing amplitude of applied vibration at fixed the frequency of applied vibration.

\section{CONCLUSION}

We can now summarize the effects of vibration on critical Marangoni number in the 
frequency range $0.4-100 \mathrm{~Hz}$. Combining the values of critical Marangoni number in this range (see Fig. 7), we find that the critical Marangoni number decreases with increasing frequency when this frequency is lower than the frequency of its internal hamonic oscillation. Futhermore for intermediate frequency, the critical Marangoni number will increase with increasing vibration frequency. The critical Marangoni number has a sudden drop near $13 \mathrm{~Hz}$. In this frequency range, the change of the shape of the free snrface in response to vibration is sharp and disordered. It may be the result of a resonance effect between vibrator and the liquid bridge. At high frequencies, the critical Marangoni number will decrease with increasing frequency of applied vibration contrary the intermediate frequency case. The frequency at which the critical Marangoni number is maximum will be influenced by the fixed amplitude of applied vibration.

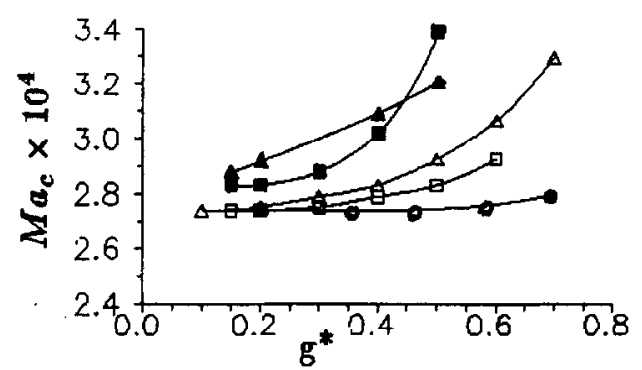

Fig. 6 Relation between the critical Marangoni number and the amplitude of vibration at fixed frequency. Here

$\Delta: f=20 \mathrm{~Hz}, \mathrm{G}: \mathrm{f}=25 \mathrm{~Hz}, \Delta: \mathrm{f}=40 \mathrm{~Hz}$,

$\square: \mathrm{f}=50 \mathrm{~Hz}, \mathrm{O}: \mathrm{f}=\mathbf{1 0 0 \mathrm { Hz }}$

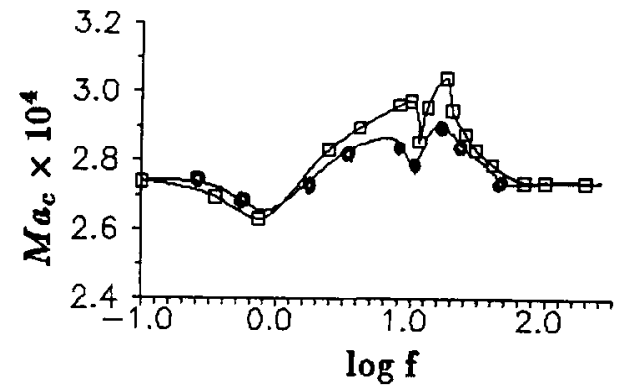

Fig. 7 Relation between the critical Marangoni number and the frequency of vibration in whole frequency range. Here $O: \mathrm{g}^{*}=0.16, \square: \mathrm{g}^{*}=0.25$

The shape change of the free surface can affect the shearing action in the Marangoni boundary layer which can influence the temperature distribution and intensity and structure of thermocapillary flows. All these may influence the onset of thermocapillary oscillation. Vibrtaion will inflnence the shape of free surface in liquid bridge. The response of free surface shape is determined by the strength, frequency and dirction of vibration. Our experiment implies that the vibration with low frequency will be harmful for floating zone processing. When the frequency is high enough, the effects of vibration are insignificant. The effects of vibration can increase the stability of thermocapillary convection in proper frequency range influenced by strength of 8 -jitter. Therefore the control of thermocapillary convection by vibration of proper strength, frequence and dirction should be taken into account for space technologies.

\section{REFEREENCE}

1. Ghun, C.H. and Wuest, W., Aeta Astronautica, 6 (1979), 1073

2. Tang, Z.M. and Hu, W.R, Microgravity Quarterly, No.3 (1992)

3. R. Monti and R. Fortezza.,Mierogravity Quarterly, 2 (1991), 163

4. Hu. W.R and Tang. Z.M, Science in Chine, 33 (1990), 934

5. Ostrach, S., and Kamotanï, Y. and Lai, C. L. ,PHY Physicochemical Hydrodynamic s, 6 (1985), 585

6. Baugher, C.R., and Martin, G.L NASA Technical Memorandum 106059

7. Alexander, J.I.D., Mierogravity Sei. Technol, vol. 4, No. 2 (1991)

8. H. Tang, F. Lu, W.R. Hn, Microgravity Sci. Technol, Vol. 7 No. 2, (1994)

9. H. Tang, W.R. Hu, Microgravity Sei. Technol, in press.

10. Z.H. Gao, et. al, Science in China, 6 (1992), 35. 